\title{
An Edge Detection Method for Suspicious Local Regions in CT images with Jaxtapleural Nodules
}

\author{
Changli Feng ${ }^{1, \mathrm{a}}$, Haiyan $\mathrm{Wei}^{2}$, Min $\mathrm{Li}^{3}$, Xin $\mathrm{Li}^{1}$ and Min Ding ${ }^{2}$ \\ ${ }^{1}$ Department of Information Science and Technology, Taishan University, 271021 Taian, China \\ ${ }^{2}$ Department of Teachers and Education, Taishan University, 271021 Taian, China \\ ${ }^{3}$ Imaging Department, Tai'an No.1 People's Hospital, Taian 271021, China
}

\begin{abstract}
Juxtapleural lung nodules are often excluded from the lung region in many CT image processing algorithms which are based on intensity information. For solving this problem, a suspicious edge line detection algorithm is proposed to obtain the edge line of the suspicious local lung region in this manuscript. Firstly, the lung region in the CT image is extracted by a fixed threshold. Then a SIFT algorithm is used to detect the feature point in the lung region. To filter out the useless feature points, a closest point matching method is used. Then a K-mean method is introduced to divide those feature points into several parts in which the edges of juxtapleural Lung nodules are contained. Experiments over CT slices show that the proposed method has a great performance in detecting the edge line of suspicious regions.
\end{abstract}

\section{Introduction}

In Lung cancer CAD system, extracting the lung region in CT slices is a primary problem. For the significant difference in $\mathrm{CT}$ value between the lung region and other organs, common algorithms are designed with the help of this intensity information.

However, these difference may be less significant in some position of lung region, for example, peripheral juxtapleural lung nodules. For juxtapleural lung nodules, there is similar intensity with surrounding organs. So some lung extracting algorithms often get a non-ideal result when extracting the lung region in CT slices. As a result, it is often seen that some juxtapleural nodules regions are excluded from the lung region when using intensity information based algorithms. Thus it can be seen that several algorithms([1],[2],[3]) have been developed for automatic lung field segmentation in volumetric CT images, however, only some algorithms can deal with the juxtapleural nodules and pulmonary

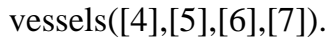

According to the words of doctors in imaging departments, when determining whether one nodule is a lung cancer, those juxtapleural Lung nodules have a higher probability than other inner isolate lung nodules. So excluding juxtapleural Lung nodules from the lung region will affect the accuracy and the performance of the CAD system greatly. For solving this problem, designing an algorithm to re-include those juxtapleural nodules region into lung region is a natural idea. And that is the reason we select this problem as the main research theme in this manuscript.

To locate the position of juxtapleural Lung nodules, many experts propose many useful algorithms. Keshani et al.([8]) proposes a method which using two morphology windows of different sizes to do a morphological operation for image enhancement. This method can void detecting the juxtapleural lung nodules and re-include all concave region into lung region again. However for the diversity of size and morphology in lung nodules, selecting an appropriate morphology template is a difficult work in practical work([9]).

Yim et al.([10]) hypothesizes that the edge section whose curvature abruptly changes is the location of juxtapleural lung nodules. This method can work well when there is no noise point on the edge line.

$\mathrm{Pu}$ et al.([11]) introduces a non-adaptive border marching algorithm to detect the convex tracks of the borderline. As what is mentioned in that manuscript, the lung border is designed as a data set of line segments. According to the relationship between the remaining points, determining whether the local region is recognized as a juxtapleural nodule region.

Messay et al.([12]) use 8 control points to obtain a more consistent lung segmentation result. Wei et al.([5]) propose the chain code method to mark the convexconcave points in the borderline. Dai et al.([13]) utilize Graham scanning method to get the convex point set, and then according to the cross product result to decide the local region is convex or concave. Then filling those concave region with convex hull method. Singadkar et al.([14]) utilize the curvature of the binary mask of the lung region, which is obtained after border smoothing, to determine the exact peak or valley points. Yuan et al.([15]) model the boundary points as a point set, and decide whether a point is concave according to the inner product of adjacent straight line section.

a Corresponding author: tafchl@tsu.edu.cn 
In this manuscript, we propose a new method which can detect the edge line of the suspicious local lung region, which contains juxtapleural nodules. In section 2 , the method will be proposed, and the main step and algorithm design will be described in this section too. In section 3, we will operate some experiments of the proposed algorithm on the several slices of CT images. In Section 4, the main contents of this manuscript will be summarized.

\section{An Edge Detection Method For Suspicious Local Regions}

\subsection{Main Steps of Algorithm}

In this section, all the frames of the proposed algorithm will be presented one by one in the following sub-section. The input data of the proposed model is an initial CT image. Then the mask of lung region will be extracted from input data through a lung region segmentation method. In the next step, the SIFT points are detected which are located in the edge line mostly. In the following, the clustering analysis method was used to divide those SIFT points into several parts. In order to deal with classification error in the above result, the optimization step was introduced. In the end, the suspicious edge section with different types are detected.

The block diagram for every steps of the proposed method in this manuscript is shown in Fig. 1.

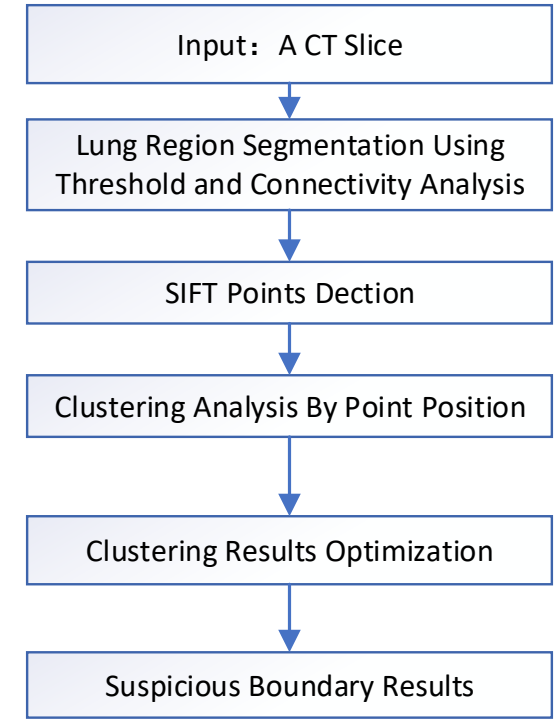

Figure 1. Main Steps of the Proposed Model

\subsection{Lung Region Segmentation}

To achieve higher segmentation velocity, a fixed threshold is selected to acquire a binary image from a CT slice. In this manuscript, the fixed threshold is set to be $550 \mathrm{HU}$ according to other works and expert advice. Then the connectivity Analysis is operated in the binary image. In order to get a hole-free lung region, the hole filling method is used to fill the small hole in the lung region which corresponding to the blood vessel or lung nodules.

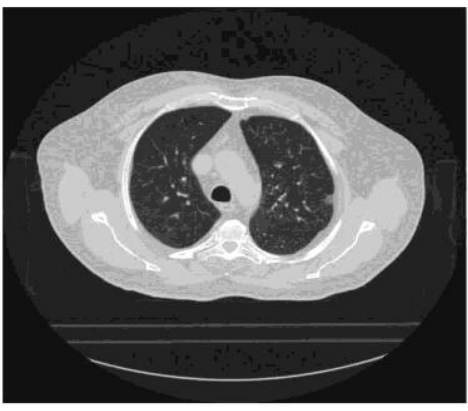

(i)

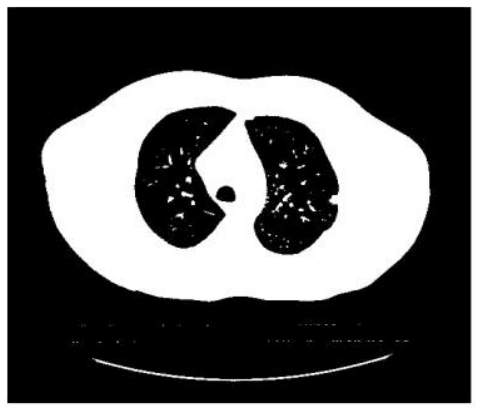

(ii)

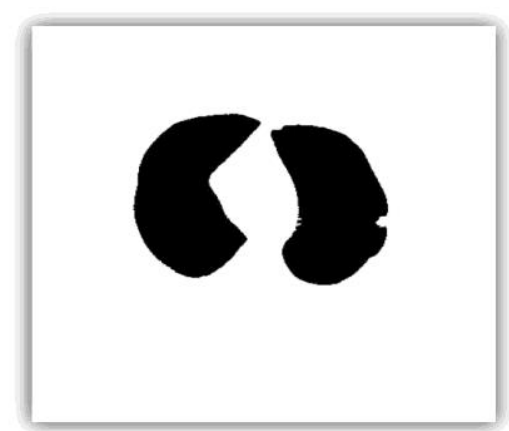

(iii)

Figure 2. The main results of step 2.2. (i)The original CT image; (ii)The binary image; (iii)The initial hole-free lung region.

\subsection{SIFT Feature Points Detection}

When getting the above initial lung region, it is easy to find that there is an obvious concave region in the right lung edge in Fig. 2(iii). At the same time, according to medical knowledge, this concave region is the juxtapleural nodules with larger probability. Thus in this subsection, the method that can detect all the feature points in the lung edge is introduced. The used method is the SIFT algorithm, it can be formulated as follows: firstly, a scale space of an image is defined as

$$
\mathrm{L}(\mathrm{x}, \mathrm{y}, \sigma)=G(x, y, \sigma) I(x, y)
$$

where ${ }^{G(x, y, \sigma)=\frac{1}{2 \pi \sigma^{2}} e^{-\frac{(x x+y 2)}{2 \sigma}}}$ is a Gaussian function with a variable parameter $\sigma$. Secondly, difference operation of Gaussians is played in the scale space to obtain the extreme points, the operation can be formulated as $\overline{\mathrm{D}}(\mathrm{x}, \mathrm{y}, \sigma)=\overline{(\mathrm{G}}(\mathrm{x}, \mathrm{y}, \mathrm{k} \sigma)-\mathrm{G}(\mathrm{x}, \mathrm{y}, \sigma)) \mathrm{I}(\mathrm{x}, \mathrm{y})$

When removing the unstable edge noisy points, selecting a $16 \times 16$ pixels neighbour window of the key point. After the histogram is calculated, accumulating the value of every direction. As a result, the sift feature point is generated which can be seen in Fig. 3(i).

However, it is worth noting that some SIFT points are not in the lung region of the Lung region. At the same time, the outside region of lung region is useless 
for our work, thus those points need to be mapped into lung region in the next operation.

So according to the above analysis, the closest mapping method is presented to do this work. Suppose a point $p_{i}^{\prime}$ is one of points in $S_{f}=\left\{p_{1}^{\prime}, p_{2}^{\prime}, p_{3}^{\prime}, \cdots, p_{m}^{\prime}\right\}$ and $S_{f}$ are the set of all feature points that are outside of lung region, the set $S_{e}=\left\{p_{1}, p_{2}, p_{3}, \cdots, p_{n}\right\}$ is all the edge line points set of the lung region. To take full advantage of the SIFT feature, the point $p_{i}^{\prime}$ in $S_{f}$ is mapped to the closest point $p_{k}$ in set $S_{e}$. The mapping method can be expressed as

$$
p_{k}=\underset{p_{i} \in S_{e}}{\arg \min _{e}}\left(\sqrt{\left(p_{i_{x}}-p_{i_{x}}^{\prime}\right)^{2}+\left(p_{i_{y}}-p_{i_{y}}^{\prime}\right)^{2}}\right) \text {. }
$$

When all points in $S_{f}$ are mapped to its corresponding edge point, we can get the result as what is shown in Fig. 3(ii).
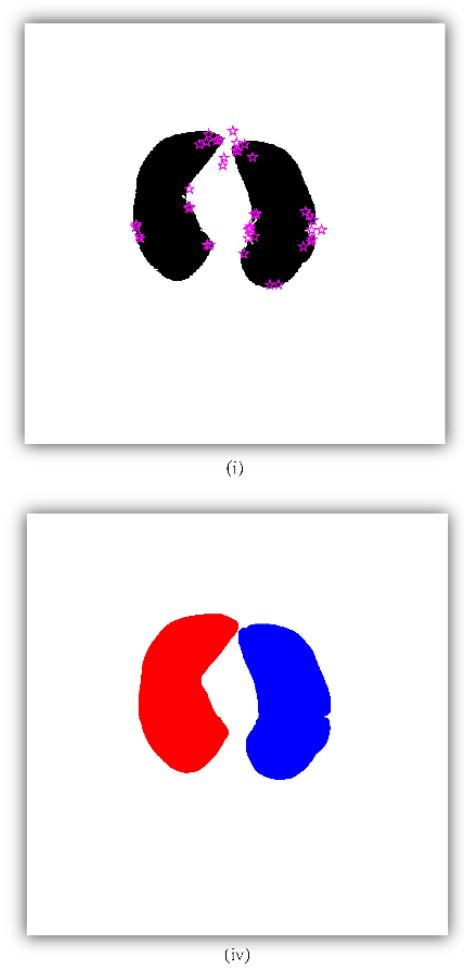

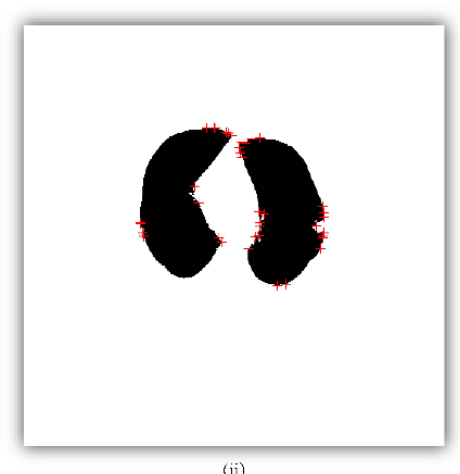

(ii)

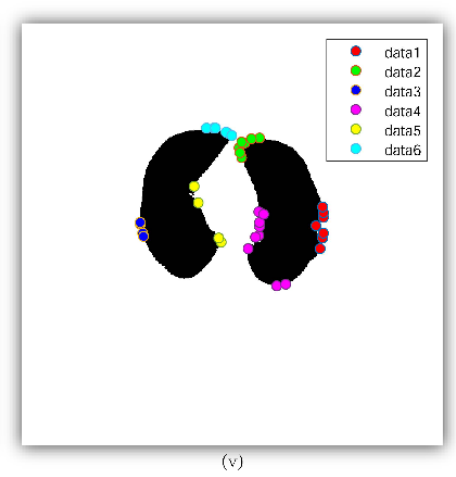

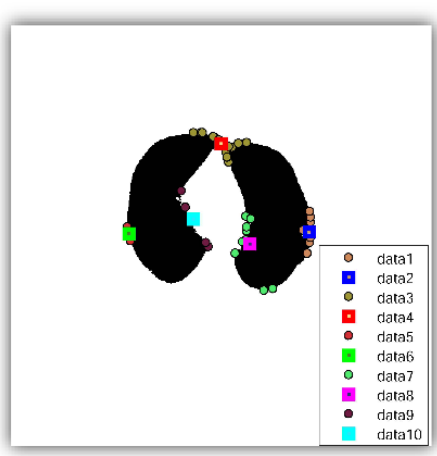

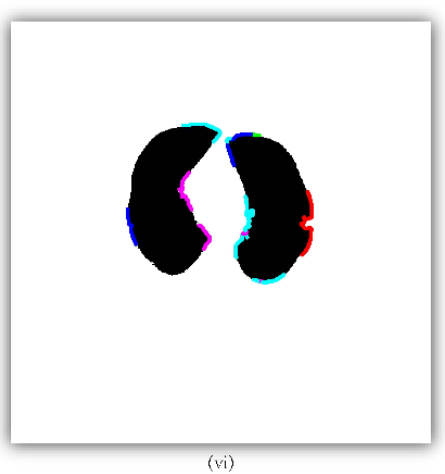

Figure 3. The results of the proposed method in this manuscript. (i) The detected SIFT feature points; (ii)The closest distance mapping points of the SIFT feature points; (iii) The initial clustering analysis results; (iv)The classification result of left and right lung sub-region; (v)The optimized classification result; (vi)The final suspicious lung edge line;

\subsection{Clustering Analysis Method}

When the sift points are calculated, the clustering analysis method is used to divide those sift into several parts. The distance between points are selected to be the Euclidian distance which can be formulated as

$$
\mathrm{d}\left(I_{1}\left(x_{1}, y_{1}\right), I_{2}\left(x_{2}, y_{2}\right)\right)=\sqrt{\left(x_{1}-x_{2}\right)^{2}+\left(y_{1}-y_{2}\right)^{2}}
$$

In the clustering analysis method, suppose there are $\mathrm{K}$ clustered types $\mathrm{S}=\left\{S_{1}, S_{2}, S_{3}, \cdots, S_{K}\right\}$. For a given observation object $\left(x_{1}, x_{2}, x_{3}, \ldots, x_{n}\right)$, there are $d$ observation parameters in every object, it can be seen as a d-dimensional vector in a data space. Then the used Kmeans clustering method can be written as

$$
\arg \min _{S} \sum_{i=1}^{K} \sum_{x \in S_{i}}\left\|x-\mu_{i}\right\|^{2}
$$

where $\mu_{i}$ means the mean value of all points in the type $S_{i}$.
The main steps of the clustering analysis method can be summarized as follows.

\section{Clustering Algorithm:}

(1) All sample points are classified into K initial types.

(2) Every point is assigned into one type from which the point has the closest distance one by one, then calculating the center of changed types again.

(3) Go to Step.(2) until all types are not changed yet.

When the algorithm executing is finished, the final classification result is got. As a result, all SIFT feature points are classified into 4-6 types generally. Besides, the number of the types are determined according to the data information, which can be set an optimal value with the help of the clustering algorithm. The result of this step can be seen in the Fig. 3(iii).

\subsection{Clustering Results Optimization}

From the Fig. 3(iii), it is easy to find that there is some classification error. Especially, on the top of lung region, 
some points which belong to left lung sub-region and right lung sub-region respectively are classified into the same type by mistake. Thus, it is necessary to optimize those classification result.

In order to avoid the above error, the lung region needs to be labeled two sub-parts: left sub-region and right sub-region. It is worth to note that one type points in ideal classification result should belong to left or right sub-region. If one type contains both left sub-region points and region sub-region, that is a wrong answer.

Firstly, as Fig. 3(iv), the clustering analysis method is used again to divide all lung region points into two parts: left and right. And points belong to the left sub-region are labeled as the value 1, points belong to the right subregion are labeled as the value 2 .

Secondly, for each type of the clustering result of Method 2.4, checking whether there are both left subregion points and right sub-region points. If the answer is true, all SIFT points that belong to this type are divided into two parts again according to their label (the label is 1 or 2). The optimized result is placed in Fig. 3(v).

In the end, as what can be seen in Fig. 3(vi), all the neighborhood points of those SIFT points in every type are set to be the suspicious edge line. The line will be analyzed in the future works to recognize whether they are on the edge of Juxtapleural nodules.

\section{Experiment}

For testing the performance of the proposed method, some experiments are operated on the CT images which are chosen from the LIDC database. There are one or two Jaxtapleural nodules in those CT images. The experiment results are shown in Fig. 4, from which it is easy to find that the proposed method in this manuscript has well performance in detecting edge lines of the suspicious points in the $\mathrm{CT}$ image.

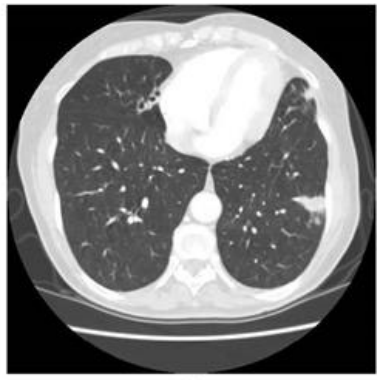

(i)

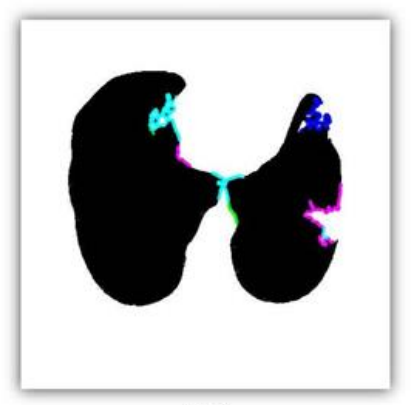

(iv)

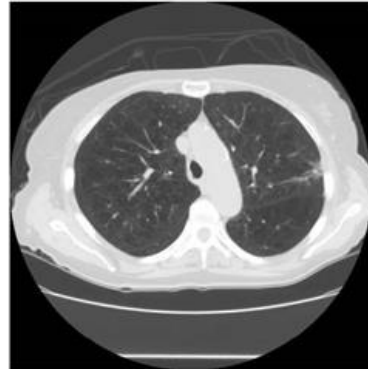

(ii)

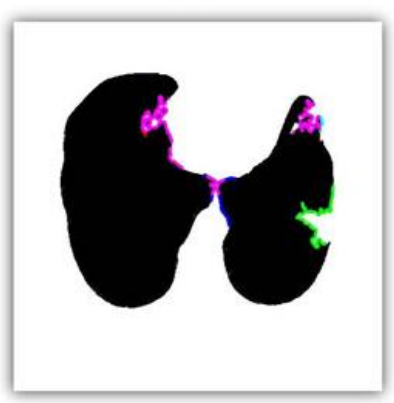

(v)

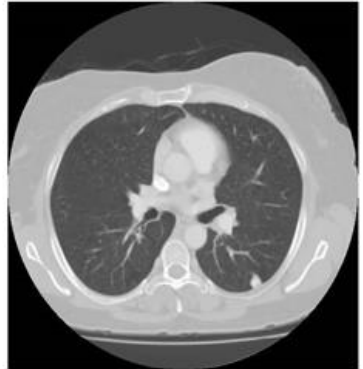

(iii)

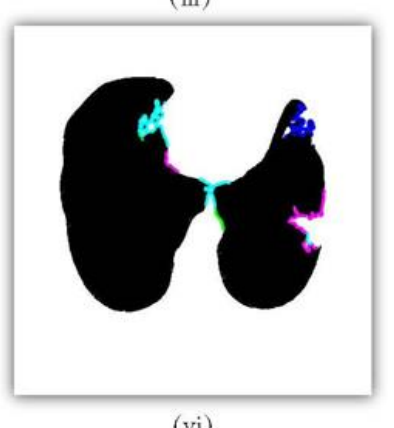

(vi)

Figure 4. Experiments on CT images. (i) (iii) An original CT image with a juxtapleural nodule. (iv) (vi) The detected suspicious edge line for images (i) (iii) correspondingly.

\section{Summary}

In this manuscript, a method that can detect the edge line of the suspicious points in a CT image is presented. This method can be described as five main steps: 1. Lung initial region extraction; 2. SIFT points detecting; 3 . Clustering analysis and classification; 4. Classification result optimization; 5. Edge line calculation. Through experiments on CT images, the proposed method is proved to be a good performance on detecting the edge line of the suspicious points in the CT image.

\section{ACKNOWLEDGE}

This work is supported by the Science and Technology Development Program of Tai'an 2017, the Scientific Research Fund of Taishan University 2014, Young
Teacher Research Fund Project of Taishan University 2017.

\section{References}

1. Sun, C. Bauer, R. Beichel. Automated 3-D segmentation of lungs with lung cancer in ct data using a novel robust active shape model approach. IEEE Trans. Med. Imaging. 31 (2): 449-460(2012)

2. A. Mansoor, U. Bagci, Z. Xu, B. Foster, K.N. Olivier, J.M. Elinoff, A.F. Suffredini, J.K. Udupa, D.J. Mollura. A generic approach to pathological lung segmentation. IEEE Trans. Med. Imaging. 33 (12):2293-2310(2014)

3. I. Sluimer, A. Schilham, M. Prokop, B. van Ginneken. Computer analysis of computed 
tomography scans of the lung: a survey. IEEE Trans. Med. Imaging. 25 (4):385-405(2006)

4. S. Shen, A.A. Bui, J. Cong, W. Hsu. An automated lung segmentation approach using bidirectional chain codes to improve nodule detection accuracy. Comput. Biol. Med. 57:139-149(2015)

5. Wei Y, Shen G, Li J J. A fully automatic method for lung parenchyma segmentation and repairing. Journal of Digital Imaging. 26(3): 483-495(2013)

6. S. Zhou, Y. Cheng, S. Tamura. Automated lung segmentation and smoothing techniques for the inclusion of juxtapleural nodules and pulmonary vessels on chest ct images. Biomed. Signal Process. Control. 13:62-70(2014)

7. A.A. Farag, H.E.A.E. Munim, J.H. Graham, A.A. Farag. A novel approach for lung nodules segmentation in chest CT using level sets. IEEE Trans. Image Process. 22:5202-5213(2013)

8. M. Keshani, Z. Azimifar, F. Tajeripour, et al. Lung nodule segmentation and recognition using SVM classifier and active contour modeling: A complete intelligent system. Computers in biology and medicine. 43(4): 287-300(2013)

9. D. Kim, J. Kim, S. Noh, et al. Pulmonary nodule detection using chest CT images. Acta Radiologica. 44(3): 252-257(2003)

10. Yim Y, Hong H. Correction of segmented lung boundary for the inclusion of pleural nodules and pulmonary vessels in chest CT images. Computers in biology and medicine. 38(8): 845-857(2008)

11. $\mathrm{Pu} \mathrm{J,} \mathrm{Roos} \mathrm{J,} \mathrm{Chin} \mathrm{A} \mathrm{Y,} \mathrm{et} \mathrm{al.} \mathrm{Adaptive} \mathrm{border}$ marching algorithm: automatic lung segmentation on chest CT images. Computerized Medical Imaging and Graphics. 32(6): 452-462(2008)

12. Messay T, Hardie R C, Tuinstra T R. Segmentation of pulmonary nodules in computed tomography using a regression neural network approach and its application to the lung image database consortium and image database resource initiative dataset. Medical Image Analysis. 22(1): 48-62(2015)

13. Shuangfeng Dai, Ke Lü, Rui Zhai, Jiyang Dong. Lung Segmentation Method Based on 3D Region Growing Method and Improved Convex Hull Algorithm., Journal of Electronics and Information Technology. 38(9): 2358-2364(2016)

14. Ganesh Singadkar, Abhishek Mahajan, Meenakshi Thakur, Sanjay Talbar. Automatic lung segmentation for the inclusion of juxtapleural nodules and pulmonary vessels using curvaturebased border correction. Journal of King Saud University - Computer and Information Sciences. 2018(2018)

15. Yuan Kehong, Xiang Lanxi. Automated lung segmentation for chest CT images used for computer-aided diagnostics. Journal of Tsinghua University. 51(1): 90-95(2011) 